\title{
Dihydrophenanthrenes from Bletilla formosana
}

\author{
Yun-Lian Lin, ${ }^{*}$ Wan-Ping Chen, and Abdulgafor Datu Macabalang \\ National Research Institute of Chinese Medicine; Taipei 112, Taiwan. Received March 14, 2005; accepted May 27, 2005
}

Three new dihydrophenanthrenes, 4-methoxy-9,10-dihydrophenanthrene-1,2,7-triol (1), 1-(4-hydroxybenzyl)-4,7-dimethoxy-9,10-dihydrophenanthrene-2-ol (2), and 1,3,6-tri(4-hydroxybenzyl)-4-methoxydihydrophenanthrene-2,7-diol (3) together with seven known phenanthrene derivatives, six known flavonoids, a bibenzyl and three phenolic compounds were isolated from the whole plant of Bletilla formosana. Their structures were elucidated by spectroscopic, mainly 2D NMR spectrometry and chemical methods.

Key words Bletilla formosana; phenanthrenes; flavonoids; bibenzyl

Bletilla formosana HAYATA (Orchidaceae) is a perennial herb with rhizomes, corms, or root-stem tuberoids. It is the only variable species of Bletilla in Taiwan. ${ }^{1)}$ The tubers of Bletilla striata ReICHB. FIL. has attracted much attention during the Severe Acute Respiratory Syndrome (SARS) prevalent period due to its use in treating pneumonophthisis and pneumonorrhagia in traditional Chinese medicine. ${ }^{2)}$ To the best of our knowledge, there has been no phytochemical investigation on $B$. formosana. As part of our effort to search for bioactive components from local indigenous herbs, we describe herein the isolation of three new dihydrophenanthrenes, along with seven known phenanthrene derivatives, six known flavonoids, a bibenzyl and three phenolic compounds from the whole herb of $B$. formosana.

\section{Results and Discussion}

The ethanolic extract of the whole herbs was successively partitioned with ethyl acetate and $n$-butanol. The ethyl acetate and $n$-butanol-soluble fractions were separatedly subjected to silica gel column chromatography. Fractions rich in polyphenolic compounds were combined and purified by Sephadex LH-20 column to give three new dihydrophenanthrenes, 4-methoxy-9,10-dihydrophenanthrene-1,2,7-triol (1), 1-(4-hydroxybenzyl)-4,7-dimethoxy-9,10-dihydrophenanthrene-2-ol (2), and 1,3,6-tri(4-hydroxylbenzyl)-4-methoxydihydrophenanthrene-2,7-diol (3), along with seven known phenanthrene derivatives, 1-(4-hydroxybenzyl)-4-methoxy9,10-dihydrophenanthrene-2,7-diol (4), ${ }^{3)}$ 1,6-di(4-hydroxylbenzyl)-4-methoxy-9,10-dihydrophenanthrene-2,7-diol (5), 1,3-di(4-hydroxybenzyl)-4-methoxy-9,10-dihydrophenanthrene-2,7-diol (6), 3,4) 1-(4-hydroxybenzyl)-4-methoxyphenanthrene-2,7-diol (7), ${ }^{4)}$ 1-(4-hydroxybenzyl)-4,8-di-methoxyphenanthrene-2,7-diol (8), ${ }^{5)}$ 1,8-bi(4-hydroxybenzyl)-4-methoxyphenanthrene-2,7-diol (9), ${ }^{6}$ and blestriarene B (10), ${ }^{7}$ a bibenzyl, 2',6'-bis( $p$-hydroxybenzyl)-3,3'-dihydroxy-5methoxybibenzyl (11), ${ }^{8)}$ six known flavonoids, 8-C-p-hydoxybenzylkaempferol (12), ${ }^{9}$ apigenin (13), ${ }^{10)}$ 6-methoxykaempferol (14), ${ }^{11)}$ kaempferol (15), ${ }^{12)}$ isorhamnetin (16), ${ }^{13)}$ kaempferol 7-O-glucoside (17), ${ }^{12)}$ 3,4-dihydroxybenzaldehyde (18), ${ }^{14)}$ protocatechuic acid (19), ${ }^{12)} p$-(hydroxymethyl)phenyl- $\beta$-D-glucoside (20). ${ }^{15)}$

Compound $\mathbf{1}$ was isolated as a pale yellow amorphous powder with a molecular formular $\mathrm{C}_{15} \mathrm{H}_{14} \mathrm{O}_{4}$ by high-resolution (HR)-EI-MS at $m / z$ 258.0951. UV absorption maxima at $218,268,279,297$, and 317 sh were characteristic of a dihydrophenanthrene skeleton. ${ }^{16,17)}$ The IR spectrum showed ab- sorptions at $3397(\mathrm{OH}), 1598$ and 1462 (benzenoid) $\mathrm{cm}^{-1}$. The ${ }^{1} \mathrm{H}-\mathrm{NMR}$ spectrum of $\mathbf{1}$ (see Table 1) showed a methoxyl group at $\delta 3.89$, an isolated aromatic proton at $\delta 6.57$, an ABX system aromatic protons at $\delta 8.04(\mathrm{~d}, J=8.0 \mathrm{~Hz}), 6.64$ (dd, $J=8.0,2.0 \mathrm{~Hz})$ and $6.60(\mathrm{~d}, J=2.0 \mathrm{~Hz}$ ), and signals assignable to the 9- and 10-methylene protons $[\delta 2.51$ and 2.57 ( $2 \mathrm{H}$ each, $\mathrm{m}, \mathrm{H}-9$ ); $\delta 2.28$ and 2.36 (2H each, $\mathrm{m}, \mathrm{H}-10)]$ of
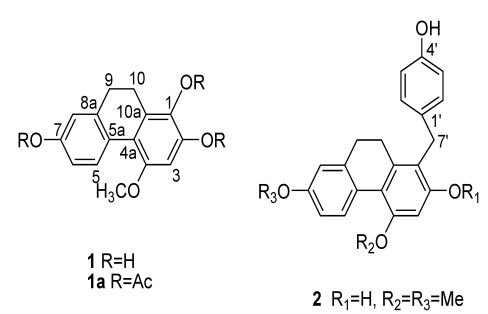
$4 \mathrm{R}_{1}=\mathrm{R}_{3}=\mathrm{H}, \mathrm{R}_{2}=\mathrm{Me}$
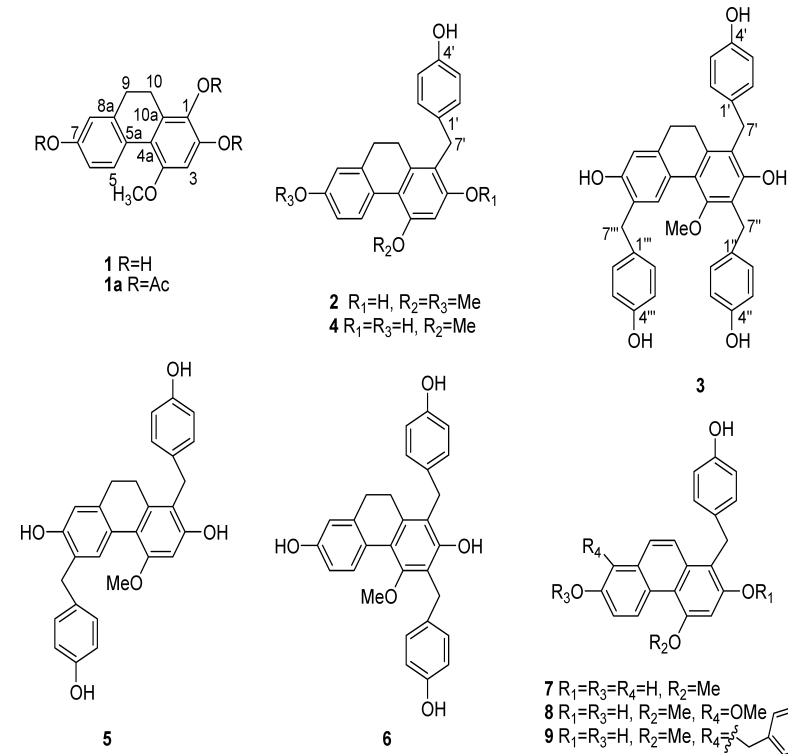

3

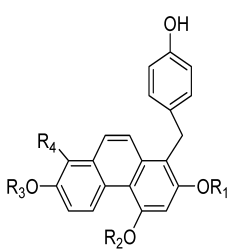

$7 \mathrm{R}_{1}=\mathrm{R}_{3}=\mathrm{R}_{4}=\mathrm{H}, \mathrm{R}_{2}=\mathrm{Me}$

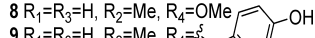

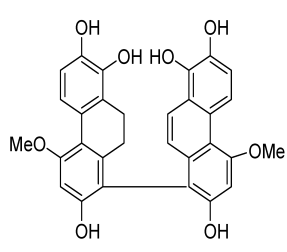

10

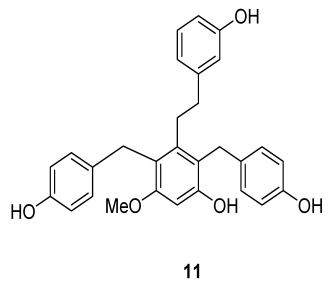

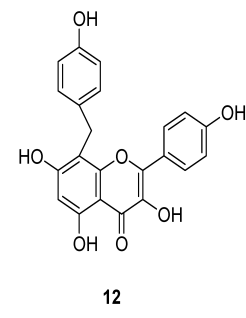

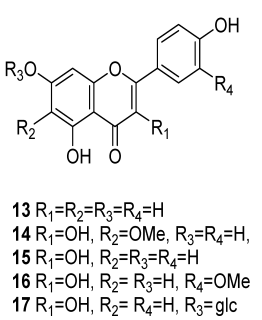

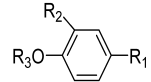

$18 \mathrm{R}_{1}=\mathrm{CHO}, \mathrm{R}_{2}=\mathrm{OH}, \mathrm{R}_{3}=\mathrm{H}$ $19 \mathrm{R}_{1}=\mathrm{COOH}, \mathrm{R}_{2}=\mathrm{OH}, \mathrm{R}_{3}=\mathrm{H}$ $20 \mathrm{R}_{1}=\mathrm{CH}_{2} \mathrm{OH}, \mathrm{R}_{2}=\mathrm{H}, \mathrm{R}_{3}=\mathrm{glc}$ 
Table 1. ${ }^{1} \mathrm{H}$ - and ${ }^{13} \mathrm{C}$-NMR Spectral Data for Compounds $\mathbf{1}, \mathbf{2}$ and $\mathbf{3}$ in $\mathrm{CD}_{3} \mathrm{OD}$

\begin{tabular}{|c|c|c|c|c|c|c|}
\hline \multirow{2}{*}{ Position } & \multicolumn{2}{|c|}{1} & \multicolumn{2}{|c|}{2} & \multicolumn{2}{|c|}{3} \\
\hline & ${ }^{1} \mathrm{H}$ & ${ }^{13} \mathrm{C}$ & ${ }^{1} \mathrm{H}$ & ${ }^{13} \mathrm{C}$ & ${ }^{1} \mathrm{H}$ & ${ }^{13} \mathrm{C}$ \\
\hline 1 & & $141.6 \mathrm{~s}$ & & $117.1 \mathrm{~s}$ & & $122.9 \mathrm{~s}$ \\
\hline 2 & & $155.4 \mathrm{~s}$ & & $156.0 \mathrm{~s}$ & & $154.2 \mathrm{~s}$ \\
\hline 3 & $6.57 \mathrm{~s}$ & $99.1 \mathrm{~d}$ & $6.52 \mathrm{~s}$ & $99.1 \mathrm{~d}$ & & $122.0 \mathrm{~s}$ \\
\hline 4 & & $158.5 \mathrm{~s}$ & & $157.3 \mathrm{~s}$ & & $156.4 \mathrm{~s}$ \\
\hline $4 \mathrm{a}$ & & $117.5 \mathrm{~s}$ & & $118.5 \mathrm{~s}$ & & $121.6 \mathrm{~s}$ \\
\hline 5 & $8.04 \mathrm{~d}(8.0)^{a)}$ & $130.3 \mathrm{~d}$ & $8.05 \mathrm{~d}(8.0)$ & $130.2 \mathrm{~d}$ & $7.96 \mathrm{~s}$ & $130.6 \mathrm{~d}$ \\
\hline $5 a$ & & $126.5 \mathrm{~s}$ & & $127.5 \mathrm{~s}$ & & $127.6 \mathrm{~s}$ \\
\hline 6 & $6.64 \mathrm{dd}(8.0,2.0)$ & $113.5 \mathrm{~d}$ & $6.71 \mathrm{dd}(8.0,2.0)$ & $112.0 \mathrm{~d}$ & & $126.2 \mathrm{~s}$ \\
\hline 7 & & $156.1 \mathrm{~s}$ & & $158.7 \mathrm{~s}$ & & $152.9 \mathrm{~s}$ \\
\hline 8 & $6.60 \mathrm{~d}(2.0)$ & $114.7 \mathrm{~d}$ & $6.70 \mathrm{~d}(2.0)$ & $113.2 \mathrm{~d}$ & $6.61 \mathrm{~s}$ & $114.6 \mathrm{~d}$ \\
\hline $8 \mathrm{a}$ & & $140.8 \mathrm{~s}$ & & $141.0 \mathrm{~s}$ & & $138.2 \mathrm{~s}$ \\
\hline 9 & $\begin{array}{l}2.51 \mathrm{~m} \\
2.57 \mathrm{~m}\end{array}$ & $31.0 \mathrm{t}$ & $2.52-2.58 \mathrm{~m}$ & $31.0 \mathrm{t}$ & $2.50 \mathrm{~m}$ & $30.8 \mathrm{t}$ \\
\hline 10 & $\begin{array}{l}2.28 \mathrm{~m} \\
2.36 \mathrm{~m}\end{array}$ & $28.2 \mathrm{t}$ & $2.52-2.58 \mathrm{~m}$ & $27.4 \mathrm{t}$ & $2.58 \mathrm{~m}$ & $27.5 \mathrm{t}$ \\
\hline $10 \mathrm{a}$ & & $115.7 \mathrm{~s}$ & & $140.3 \mathrm{~s}$ & & $138.8 \mathrm{~s}$ \\
\hline $1^{\prime}$ & & & & $133.8 \mathrm{~s}$ & & $133.1 \mathrm{~s}$ \\
\hline $2^{\prime}$ & & & $6.94 \mathrm{~d}(8.0)$ & $130.0 \mathrm{~d}$ & $6.94 \mathrm{~d}(8.0)$ & $130.0 \mathrm{~d}$ \\
\hline $3^{\prime}$ & & & $6.64 \mathrm{~d}(8.0)$ & $115.9 \mathrm{~d}$ & $6.60 \mathrm{~d}(8.0)$ & $115.9 \mathrm{~d}$ \\
\hline $4^{\prime}$ & & & & $155.8 \mathrm{~s}$ & & $156.1 \mathrm{~s}$ \\
\hline $5^{\prime}$ & & & $6.64 \mathrm{~d}(8.0)$ & $115.9 \mathrm{~d}$ & $6.60 \mathrm{~d}(8.0)$ & $115.9 \mathrm{~d}$ \\
\hline $6^{\prime}$ & & & $6.94 \mathrm{~d}(8.0)$ & $130.0 \mathrm{~d}$ & $6.94 \mathrm{~d}(8.0)$ & $130.0 \mathrm{~d}$ \\
\hline $7^{\prime}$ & & & $3.94 \mathrm{~s}$ & $30.9 \mathrm{t}$ & $4.01 \mathrm{~s}$ & $30.4 \mathrm{t}$ \\
\hline $1^{\prime \prime}$ & & & & & & $134.0 \mathrm{~s}$ \\
\hline $2^{\prime \prime}$ & & & & & $7.05 \mathrm{~d}(8.0)$ & $130.3 \mathrm{~d}$ \\
\hline $3^{\prime \prime}$ & & & & & $6.67 \mathrm{~d}(8.0)$ & $115.9 \mathrm{~d}$ \\
\hline $4^{\prime \prime}$ & & & & & & $156.3 \mathrm{~s}$ \\
\hline $5^{\prime \prime}$ & & & & & $6.67 \mathrm{~d}(8.0)$ & $115.9 \mathrm{~d}$ \\
\hline $6^{\prime \prime}$ & & & & & $7.05 \mathrm{~d}(8.0)$ & $130.3 \mathrm{~d}$ \\
\hline $7^{\prime \prime}$ & & & & & $4.02 \mathrm{~s}$ & $31.8 \mathrm{t}$ \\
\hline $1^{\prime \prime \prime}$ & & & & & & $134.4 \mathrm{~s}$ \\
\hline $2^{\prime \prime \prime}$ & & & & & $7.08 \mathrm{~d}(8.0)$ & $130.8 \mathrm{~d}$ \\
\hline $3^{\prime \prime \prime}$ & & & & & $6.68 \mathrm{~d}(8.0)$ & $116.0 \mathrm{~d}$ \\
\hline $4^{\prime \prime \prime}$ & & & & & & $156.3 \mathrm{~s}$ \\
\hline $5^{\prime \prime \prime}$ & & & & & $6.68 \mathrm{~d}(8.0)$ & $116.0 \mathrm{~d}$ \\
\hline $6^{\prime \prime \prime}$ & & & & & $7.08 \mathrm{~d}(8.0)$ & $130.8 \mathrm{~d}$ \\
\hline $7^{\prime \prime \prime}$ & & & & & $3.84 \mathrm{~s}$ & $35.7 \mathrm{t}$ \\
\hline $\mathrm{OMe}$ & $3.89 \mathrm{~s}$ & $55.9 \mathrm{q}$ & $3.77 \mathrm{~s}, 3.83 \mathrm{~s}$ & $55.6 \mathrm{q}, 55.9 \mathrm{q}$ & $3.14 \mathrm{~s}$ & $60.4 \mathrm{q}$ \\
\hline
\end{tabular}

a) Coupling constants are presented in $\mathrm{Hz}$.

dihydrophenanthrene. In the nuclear Overhauser effect (NOE) experiment, irradiation of the methoxyl signal caused an NOE enhancement of the signals at $\delta 6.57(\mathrm{H}-3)$ and $\delta$ 8.04 (H-5). These revealed that the methoxyl functionality is attached to $\mathrm{C}-4$. The acetylation of 1 give a triacetate $[\delta 1.92$, 2.28 and $2.28(3 \mathrm{H}$ each, s)]. Irradiation of the methyl proton at $\delta 1.92$ enhanced the signal of $\mathrm{H}-3(\delta 6.73)$. In turn, irradiation of the methyl proton at $\delta 2.28$ caused the enhancement of the signals at H-6 $[\delta 6.98(\mathrm{dd}, J=8.0,2.0 \mathrm{~Hz})], \mathrm{H}-8[\delta$ $6.92(1 \mathrm{H}, \mathrm{d}, J=2.0 \mathrm{~Hz})]$, and $\mathrm{H}-10[\delta 2.35(2 \mathrm{H}, \mathrm{m})]$. From above results, the structure of compound 1 was established as 4-methoxy-9,10-dihydrophenanthrene 1,2,7-triol.

Compound $\mathbf{2}$ was obtained as a pale yellow amorphous powder. The molecular formula of $\mathbf{2}$ was established as $\mathrm{C}_{23} \mathrm{H}_{22} \mathrm{O}_{4}$ by HR-EI-MS. It showed a significant fragment peak at $\mathrm{m} / \mathrm{z} 256$ and 107 due to the removal of hydroxybenzyl $\left(\mathrm{C}_{7} \mathrm{H}_{7} \mathrm{O}\right)$ moiety in mass spectrum (EI-MS). The IR and UV spectra were similar to those of compound 1. The ${ }^{1} \mathrm{H}$ and ${ }^{13} \mathrm{C}-\mathrm{NMR}$ spectra (see Table 1) of $\mathbf{2}$ showed similar peaks with that of 1 with the addition of a methoxy $\left(\delta_{\mathrm{H}} 3.77\right.$, $\left.\mathrm{s} ; \delta_{\mathrm{C}} 55.6 \mathrm{q}\right)$ and a $p$-hydroxybenzyl group $\left[\delta_{\mathrm{H}} 6.94\right.$ and 6.64
( $2 \mathrm{H}$ each, d, $J=8.0 \mathrm{~Hz}$ ), and $3.94(2 \mathrm{H}, \mathrm{s}) ; \delta_{\mathrm{C}} 133.8(\mathrm{~s}), 130.0$ (d), 115.9 (d), 155.8 (s), 115.9 (d), 130.0 (d), 30.9 (t)]. In the NOE experiment, irradiation of methoxy signal at $\delta 3.77$ enhanced the proton signals at $\delta 6.71(\mathrm{H}-6)$ and $\delta 6.70(\mathrm{H}-8)$, while irradiation of the methoxy signal at $\delta 3.83$ enhanced the proton signals at $\delta 6.52(\mathrm{H}-3)$ and $\delta 8.05(\mathrm{H}-5)$, and the irradiation of $\mathrm{H}^{-7}{ }^{\prime}(\delta 3.94)$ induced the enhancement of $\mathrm{H}$ 10. These confirmed the positions of the two methoxyls at $\mathrm{C}$ 4 and $\mathrm{C}-7$, and the $p$-hydroxybenzyl group at C-1. Analysis of long-range correlations in $\mathrm{HMBC}$ spectrum from $\mathrm{H}-7^{\prime}$ to $\mathrm{C}-1, \mathrm{C}-2$, and $\mathrm{C}-10 \mathrm{a}$, which allowed the connection of $p$-hydroxybenzyl to $\mathrm{C}-1$. Comparison of the ${ }^{1} \mathrm{H}$ - and ${ }^{13} \mathrm{C}-\mathrm{NMR}$ data of $\mathbf{2}$ are similar to those of 1-(4-hydroxybenzyl)-4methoxy-9,10-dihydrophenanthrene-2,7-diol ${ }^{4}$ except for a methoxyl group at 7-position in place of a hydroxyl group Therefore, the structure of compound 2 was assigned as 1-(4hydroxybenzyl)-4,7-dimethoxy-9,10-dihydrophenanthrene-2ol.

Compound 3 has the molecular formula $\mathrm{C}_{36} \mathrm{H}_{32} \mathrm{O}_{6}$ as determined by HR-EI-MS. The IR and UV spectra of $\mathbf{3}$ were similar to those of $\mathbf{1}$ and $\mathbf{2}$. The mass spectrum of $\mathbf{3}$ exhibited a 
$\left[\mathrm{M}^{+}\right]$at $\mathrm{m} / \mathrm{z} 560$ and a prominent peak at $\mathrm{m} / \mathrm{z} 256$ and 107 due to the removal of three hydroxybenzyl $\left(3 \times \mathrm{C}_{7} \mathrm{H}_{6} \mathrm{O}\right)$ groups. The ${ }^{1} \mathrm{H}$ - and ${ }^{13} \mathrm{C}$-NMR of $\mathbf{3}$ showed three $p$-hydroxybenzyl, two isolated aromatic protons, two methylene protons, and a methoxyl group. The NOE correlations between the methoxyl signal $(\delta$ 3.14) and H-5 ( $\delta$ 7.96) and benzylic methylene at $\delta 4.02$ (H-7"), between the benzylic methylene at $\delta 4.01\left(\mathrm{H}-7^{\prime}\right)$ and $\mathrm{H}-10$ and $\mathrm{H}-2^{\prime}\left(6^{\prime}\right)$, between the benzylic methylene at $\delta 3.84$ and H-5 ( $\delta 7.96)$ and H-2"' $\left(6^{\prime \prime \prime}\right)$ indicated the methoxyl group at $\mathrm{C}-4$ and the locations of three $p$-hydroxybenzyl at C-1, C-3 and C-6. The methoxyl protons in 3 was seen at higher field ( $\delta 3.14)$ due to shielding by the 3-hydroxybenzyl group. The HMBC correlations between $\mathrm{H}$ 5 and C-4a, C-5a, C-8a, C-6, C-7, and C-7"'; between H-8 and C-5a, C-6, C-7, C-8a, and C-9, between H-7" and C-2, $\mathrm{C}-3$, and $\mathrm{C}-4$; between $\mathrm{OMe}$ and $\mathrm{C}-4$ further supported the assignment of $\mathbf{3}$ as the proposed structure.

\section{Experimental}

General Experimental Procedures Melting points were determined on Yanagimoto micromelting point apparatus and are uncorrected. IR spectra were recorded on a Nicolet avatar 320 FT-IR spectrophotometer. UV spectra were measured on a Hitachi U-3310 spectrophotometer. NMR experiments were run on a Varian unity INOVA-500 spectrometer. Mass spectra (EI-MS and HR-EI-MS) were taken on JEOL JMS-HX110 and a JEOL SX-102A mass spectrometer, respectively. Column chromatography was performed on Silica gel and Sephadex LH-20 (Pharmacia). Si gel 60F ${ }_{254}$ (Merck) was used for TLC with $\mathrm{MeOH}: \mathrm{CHCl}_{3}(5: 95$ or $10: 90)$ as developing solvent.

Plant Material The whole plant of $B$. formosana was collected from the mountain area of Taipei county, in June, 2003. The plant was identified by Mr. Jun-Chih Ou, a previous associate research fellow of National Research Institute of Chinese Medicine, and through comparison with the voucher specimens already deposited at the Herbarium of the Department of Botany, National Taiwan University, Taipei, Taiwan.

Extraction and Isolation The ground air-dried whole plant of $B$. formosana $\left(20 \mathrm{~kg}\right.$ ) were extracted with EtOH (each $90 \mathrm{ml} \times 3$ ) at $60^{\circ} \mathrm{C}$ (overnight). The EtOH extracts were combined and evaporated under reduced pressure to give $c a .545 \mathrm{~g}$ residue. The concentrate was taken up in $\mathrm{H}_{2} \mathrm{O}$, and partitioned with ethyl acetate and $n$-butanol (each $21 \times 3$ ) sequentially. After evaporation, the ethyl acetate $(85 \mathrm{~g})$ and $n$-butanol $(135 \mathrm{~g})$ soluble extracts were separatedly subjected to column chromatography over silica gel (using hexane-EtOAc- $10 \% \mathrm{MeOH} / \mathrm{EtOAc}$ gradient for ethyl acetate extract, and $25 \% \mathrm{EtOAc} /$ hexane-EtOAc- $20 \% \mathrm{MeOH} / \mathrm{EtOAc}$ for $n$-butanol extract). The fractions (EtOAc to $10 \% \mathrm{MeOH} / \mathrm{EtOAc}$ ) rich in polyphenolic compounds were combined and rechromatographed over Sephadex LH-20 column with $\mathrm{MeOH}$ as eluent to yield five fractions. Each fraction was further purified on a Sephadex LH-20 column with $\mathrm{MeOH}$ as eluent repeatedly, to afford $\mathbf{1}(105 \mathrm{mg}), \mathbf{2}(35 \mathrm{mg}), \mathbf{3}(18 \mathrm{mg}), \mathbf{4}(86 \mathrm{mg}), \mathbf{5}(31 \mathrm{mg}), \mathbf{6}(28 \mathrm{mg})$, $\mathbf{7}(22 \mathrm{mg}), \mathbf{8}(19 \mathrm{mg}), \mathbf{9}(7 \mathrm{mg}), \mathbf{1 0}(8 \mathrm{mg}), \mathbf{1 1}(16 \mathrm{mg}), \mathbf{1 2}(36 \mathrm{mg}), \mathbf{1 3}$ (26 mg), 14 (12 mg), 15 (48 mg), 16 (32 mg), 17 (15 mg), 18 (78 mg), 19 $(108 \mathrm{mg})$ and $\mathbf{2 0}(86 \mathrm{mg})$.

Compound 1: Pale yellow amorphous powder; IR $(\mathrm{KBr}) \mathrm{cm}^{-1}: 3397$, $1598,1577,1462,1235,1204,1167,1077,1046,978,820$. UV $\lambda_{\max }$ $(\mathrm{MeOH}) \mathrm{nm}(\log \varepsilon): 218$ (4.52), 268 (4.01), 279 (4.03), 297 (3.95), $317 \mathrm{sh}$ (3.75). ${ }^{1} \mathrm{H}-\mathrm{NMR}\left(\mathrm{CD}_{3} \mathrm{OD}, 500 \mathrm{MHz}\right)$ see Table $1 .{ }^{13} \mathrm{C}-\mathrm{NMR}\left(\mathrm{CD}_{3} \mathrm{OD}\right.$, $125 \mathrm{MHz}$ ) see Table 1. Main NOE correlations: $\mathrm{H}-9 / \mathrm{H}-8$; OMe $/ \mathrm{H}-3, \mathrm{H}-5$. HMBC correlations: OMe/C-4; H-3/C-1, -2, $-4,-4 a ; H-5 / C-4 a,-5 a,-6,-7$, $-8 \mathrm{a}$; H-6/C-5a, -5, -7, -8; H-8/C-5a, -6, -7, -8a, -9; H-9/C-5a, -8, -8a, -10, -10a; H-10/C-1, -4a, -8a, -9, -10a. EI-MS $m / z: 258\left(\mathrm{M}^{+}\right)$(100), 243 (42). HR-EI-MS $m / z: 258.0951\left(\right.$ Calcd for $\left.\mathrm{C}_{15} \mathrm{H}_{14} \mathrm{O}_{4}: 258.0892\right)$.

Acetylation of 1 A solution of $1(5 \mathrm{mg})$ in pyridine $(0.5 \mathrm{ml})$ and $\mathrm{Ac}_{2} \mathrm{O}$ $(0.5 \mathrm{ml})$ was left at room temperature overnight. The solvent and excess reagent were removed with a high-vacuum pump. Purification by preparative TLC gave 1a $(4 \mathrm{mg})$. 1a: Colorless amorphous powder. ${ }^{1} \mathrm{H}-\mathrm{NMR}\left(\mathrm{CDCl}_{3}\right.$,
$500 \mathrm{MHz}) \delta: 1.92,2.28$ and $2.28(3 \mathrm{H}$ each, s, OAc), $2.35(2 \mathrm{H}, \mathrm{m}, \mathrm{H}-10)$, $2.48(2 \mathrm{H}, \mathrm{m}, \mathrm{H}-9), 3.91(3 \mathrm{H}, \mathrm{s}, \mathrm{OMe}), 6.73(1 \mathrm{H}, \mathrm{s}, \mathrm{H}-3), 6.92(1 \mathrm{H}, \mathrm{d}$, $J=2.0 \mathrm{~Hz}, \mathrm{H}-8), 6.98(1 \mathrm{H}, \mathrm{dd}, J=8.0,2.0 \mathrm{~Hz}, \mathrm{H}-6), 8.28(1 \mathrm{H}, \mathrm{d}, J=8.0 \mathrm{~Hz}$, $\mathrm{H}-5)$. NOE correlations: OMe/H-3, H-5; OAc $(\delta 2.28) / \mathrm{H}-2, \mathrm{H}-6, \mathrm{H}-10$; OAc $(\delta 1.92) / \mathrm{H}-3$, OAc $(\delta 2.28)$.

Compound 2: Pale yellow amorphous powder; IR $(\mathrm{KBr}) \mathrm{cm}^{-1}$ : 3411 , $1611,1593,1509,1462,1435,1256,1225,1041,810 . \mathrm{UV}(\mathrm{MeOH}) \lambda_{\max } \mathrm{nm}$ (log $\varepsilon$ ): 220 (4.55), 265 (4.00), 279 (4.02), 300 (3.90), 318 sh (3.75). ${ }^{1} \mathrm{H}-$ NMR $\left(\mathrm{CD}_{3} \mathrm{OD}, 500 \mathrm{MHz}\right)$ see Table $1 .{ }^{13} \mathrm{C}-\mathrm{NMR}\left(\mathrm{CD}_{3} \mathrm{OD}, 125 \mathrm{MHz}\right)$ see Table 1. Main NOE correlations: OMe $(\delta 3.77) / \mathrm{H}-6, \mathrm{H}-8$; OMe $(\delta 3.83) / \mathrm{H}-$ 3, H-5; H-7'/H-10, H-2'(6'). HMBC correlations: H-3/C-1, -2, -4, -4a; H5/C-4a, -5a, -8a, -6, -7; H-6/C-5a, -5, -7, -8; H-8/C-5a, -8a, -6, -7, C-9; H9/C-5a, 8, -8a, -10, -10a; H-10/C-1, -4a, -8a, -9, -10a; OMe $(\delta 3.77) / \mathrm{C}-7$; OMe $(\delta 3.83) / \mathrm{C}-4$. EI-MS $m / z: 362\left(\mathrm{M}^{+}\right)(100), 348(80), 268(65), 256$ (95), 241 (35), 213 (26), 107 (10). HR-EI-MS m/z: 362.1434 (Calcd for $\mathrm{C}_{23} \mathrm{H}_{22} \mathrm{O}_{4}: 362.1519$ ).

Compound 3: Pale yellow amorphous powder. IR $(\mathrm{KBr}) \mathrm{cm}^{-1}: 3275$, $1614,1593,1509,1440,1230,1162,1025,989,826$. UV (MeOH) $\lambda_{\max } \mathrm{nm}$ $(\log \varepsilon): 228$ (4.55), $270 \mathrm{sh}$ (4.04), 283 (4.09), $300 \mathrm{sh}$ (3.95), $318 \mathrm{sh}$ (3.85). ${ }^{1} \mathrm{H}-\mathrm{NMR}\left(\mathrm{CD}_{3} \mathrm{OD}, 500 \mathrm{MHz}\right)$ see Table $1 .{ }^{13} \mathrm{C}-\mathrm{NMR}\left(\mathrm{CD}_{3} \mathrm{OD}, 125 \mathrm{MHz}\right)$ see Table 1. Main NOE correlations: OMe/H-5, H-7"; H-5/H-7"', OMe; H8/H-9; H-7'/H-10, H-2', H-6'; H-7"/H-2", H-6", OMe; H-7"'/H-5, H-2"', H$6^{\prime \prime \prime}$. HMBC correlations: H-5/C-4a, $-5 \mathrm{a},-6,-7,-7^{\prime \prime \prime},-8 \mathrm{a} ; \mathrm{H}-8 / \mathrm{C}-5 \mathrm{a},-6,-7$, $-8 \mathrm{a},-9$; H-9/C-5a, $-8,-8 \mathrm{a},-10,-10 \mathrm{a} ; \mathrm{H}-10 / \mathrm{C}-1,-4 \mathrm{a},-8 \mathrm{a},-9,-10 \mathrm{a} ; \mathrm{H}-7^{\prime \prime} / \mathrm{C}-2$, C-3, C-4; OMe/C-4. EI-MS m/z: $560\left(\mathrm{M}^{+}\right)$(100), 348 (80), 268 (65), 256 (95), 241 (35), 213 (26), 107 (15). HR-EI-MS m/z: 560.2246 (Calcd for $\mathrm{C}_{36} \mathrm{H}_{32} \mathrm{O}_{6}: 560.2200$ ).

Acknowledgment This work was supported by the National Science Council of the Republic of China (NSC 93-2323-B-077-002).

\section{References and Notes}

1) "Flora of Taiwan," Vol. V, 2nd ed., ed. by Editorial Committee of the Flora of Taiwan, Taipei, 1994, p. 758.

2) Chang Su New Medical College, "Dictionary of Chinese Crude Drugs," Vol. I, Shanghai Scentific Technologic Publisher, Shanghai, 1996, p. 667.

3) Bai L., Kato T., Inoue K., Yamaki M., Takagi S., Phytochemistry, 33, 1481-1483 (1993).

4) Yamaki M., Bai L., Inoue K., Takagi S., Phytochemistry, 29, 22852287 (1990).

5) Tuchinda P., Udchachon J., Khumtaveeporn K., Taylor W., Phytochemistry, 28, 2463-2466 (1989).

6) Bai L., Kato T., Inoue K., Yamaki M., Takagi S., Phytochemistry, 30, 2733-2735 (1991).

7) Yamaki M., Bai L., Inoue K., Takagi S., Phytochemistry, 28, 35033505 (1989).

8) Takagi S., Yamaki M., Inoue K., Phytochemistry, 22, 1011-1015 (1983).

9) Merghem R., Jay M., Viricel M. R., Bayet C., Voirin B., Phytochemistry, 38, 637-640 (1995).

10) Deng Y. R., Song A. X., Wang H. Q., J. Chin. Chem. Soc., 51, 629636 (2004).

11) Fuchino H., Satoh T., Tanaka N., Chem. Pharm. Bull., 44, 1748-1753 (1996).

12) Lin Y. L., Wang W. Y., Kuo Y. H., Chen C. F., J. Chin. Chem. Soc., 47, $247-251(2000)$.

13) Wolbis M., Krolikowska M., Phytochemistry, 27, 3941-3943 (1988).

14) Ahyazi B. C., Crestini C., Argyropoulos D. S., J. Agric. Food Chem., 47, 190-201 (1999).

15) Hase T., Takao H., Iwagawa T., Phytochemistry, 22, 1977-1982 (1983).

16) Tezuka Y., Ji L., Hirano H., Ueda M., Nagashima K., Kikuchi T., Chem. Pharm. Bull., 38, 629-635 (1990).

17) Lin Y. L., Huang R. L., Don M. J., Kuo Y. H., J. Nat. Prod., 63, $1608-1610$ (2000). 